\title{
Gene and protein expression in the oxaliplatin-resistant HT29/L-OHP human colon cancer cell line
}

\author{
Z. Xiang ${ }^{1,2}$, Q.J. Kang ${ }^{1}$ and X. Xiang ${ }^{1}$ \\ ${ }^{1}$ Department of Gastrointestinal Surgery, \\ The First Affiliated Hospital of Chongqing Medical University, \\ Chongqing, China \\ ${ }^{2}$ Chongqing Key Laboratory, Department of General Surgery, \\ The First Affiliated Hospital of Chongqing Medical University, \\ Chongqing, China \\ Corresponding author: Z. Xiang \\ E-mail: 13330209937@163.com
}

Genet. Mol. Res. 14 (3): 11013-11022 (2015)

Received February 15, 2015

Accepted June 18, 2015

Published September 21, 2015

DOI http://dx.doi.org/10.4238/2015.September.21.14

\begin{abstract}
Oxaliplatin (L-OHP) is one of the most commonly used anticancer drugs in adjuvant treatment of colon cancer after complete resection of the primary tumor and treatment of metastatic colorectal cancer. Cancer cells eventually become resistant to L-OHP, which diminishes its curative effect. However, the mechanism of action of L-OHP remains unknown. In this study, an L-OHP-resistant human colon cancer cell line, HT29/L-OHP, was established by gradually increasing the dose of L-OHP in culture. The expression levels of the tumor susceptibility gene 101 ( $t$ sg 101) and the TSG101 protein in HT29 and HT29/L-OHP cell lines were examined by reverse transcriptionpolymerase chain reaction and western blot analysis. In addition, the expression levels of several apoptosis-regulating protein markers were determined using immunohistochemistry-staining assays. We found that the expression of $\operatorname{tsg} 101 \mathrm{mRNA}$ and of TSG101 protein were
\end{abstract}


significantly higher in the HT29/L-OHP cell line than in its parent, HT29 (P < 0.05). In addition, the expression of multiple apoptosisregulating protein markers were significantly increased $(\mathrm{P}<0.05)$ in the HT29/L-OHP cell line. These data suggest that these markers could be useful as predictive markers for evaluating and comparing the efficacy and molecular pharmacology of chemotherapeutics.

Key words: Human colon cancer cells; Multi-drug resistance; Oxaliplatin

\section{INTRODUCTION}

Oxaliplatin (cis-[(1R,2R)-1,2-cyclohexanediamine-N,N'] oxalato (2-)-O,O' platinum; Eloxatin) is a new-generation platinum derivative bearing a 1,2-diaminocyclohexane carrier ligand with broad potent anti-proliferative activity, and is a promising therapeutic drug for the treatment of advanced colorectal cancer (Voland et al., 2006). However, its therapeutic application has been frequently limited by the eventual resistance of cancer cells to the drug, and the exact mechanism for this effect is still not well understood. Multi-drug resistance (MDR) is a major factor that leads to the failure of tumor chemotherapy (Krishna and Mayer, 2000), and is mediated by a variety of elements including cell membrane proteins, enzymes, and apoptosis-related genes. Therefore, the investigation of these adverse factors at the gene and protein levels might help us better understand the mechanism of MDR action of colorectal cancers. In this study, we assessed the MDR associated with oxaliplatin using the oxaliplatinresistant human colon cancer line, HT29/L-OHP, and determined the underlying mechanisms of oxaliplatin resistance. These could be potentially used in turn for the development of an anticancer approach to avoid the development of resistance to oxaliplatin in the clinical setting.

\section{MATERIAL AND METHODS}

\section{Reagents and antibodies}

RPMI-1640 was purchased from Gibco BRL (Gaithersburg, MD, USA), newborn calf serum was from Hangzhou Sijiqing Bio-Engineering Material Ltd. Co. (Hangzhou, China), GST- $\pi$ monoclonal antibody was from Wuhan Doctor German Biology Ltd. Co. (Wuhan, China), reagent kits for gene and protein marker assays such as MRP, NF- $\kappa B$, HSP70, iNOS, MTP53, Bcl-2, Bax, S-P, and DAB were from Beijing Cedar Jinqiao Biological Technology Ltd. Co. (Beijing, China), the antibody against TSG101 (M-19) was from Santa Cruz Biotechnology (Santa Cruz, CA, USA), nitrocellulose membranes were from Sigma-Aldrich Corp. (St. Louis, MO, USA), and oxaliplatin (L-OHP) was from Jiangsu Heng-rui Medicine Ltd. Co. (Jiangsu, China).

\section{Establishment of the HT29/L-OHP cell line}

The human colon cancer cell line HT29 was obtained from the Chongqing Key Laboratory of the Department of General Surgery, the First Affiliated Hospital of Chongqing 
Medical University. HT29 cells were seeded in a $10-\mathrm{mL}$ flask and $4 \mu \mathrm{M}\left(1 / 3 \mathrm{IC}_{50}\right) \mathrm{L}-\mathrm{OHP}$ solution was added into the medium, and then the medium was changed after a 48 -h incubation. Repeated $4 \mu \mathrm{M}$ L-OHP incubations were performed when cells recovered to normal growth and subculture. After subculture and incubation with L-OHP solution was repeated 11 times (L-OHP concentration increased by $1 \mu \mathrm{M}$ each subculture), the HT29 cell line from the last subculture, which was resistant to $15 \mu \mathrm{M}$ L-OHP, was cultured in complete culture medium containing $4 \mu \mathrm{M} \mathrm{L}-\mathrm{OHP}$ and was defined as HT29/L-OHP.

\section{MDR of the HT29/L-OHP cell line}

The resistance of HT29/L-OHP and its parent cell HT29 line to L-OHP, vincristine (VCR), fluorouracil (5-FU), doxorubicin (ADM), and mitomycin (MMC) was detected by the MTT assay. Five different concentrations of each of these five antineoplastic drugs (L-OHP: 6.3, 12.6, 25.2, 50.4, and $100.8 \mu \mathrm{M}$; VCR: 0.3, 0.6, 1.2, 2.4, and $4.8 \mu \mathrm{M}$; 5-FU: 192.2, 384.4, 766.8, 1537.5, and $3075 \mu \mathrm{M}$; ADM: 4.6, 9.2, 18.4, 36.8, and 73.6 $\mu \mathrm{M}$; MMC: 0.75, 1.5, 3.0, 6.0 , and $12.0 \mu \mathrm{M}$ ) were incubated with HT29/L-OHP and HT29 cells for $72 \mathrm{~h}$, and then MTT was added into the medium. After 4-h incubation, the supernatant was discarded and the cells were dissolved by dimethyl sulfoxide $(200 \mu \mathrm{L} /$ well). Finally, the absorbance was read by a micro-plate reader (BIO-RAD, USA) $(570 \mathrm{~nm})$. The survival rate of each concentration was calculated by the formula: survival rate $\%=$ AHT29/L-OHP/AHT29 x $100 \%$. Intermediate drawing was performed according to the efficiency formula: $\mathrm{fa} / \mathrm{fu}=(\mathrm{D} / \mathrm{Dm}) \mathrm{m}$, where $\mathrm{fu}=$ survival rate, $\mathrm{fa}=1$ - fu, $\mathrm{D}=$ drug dose, $\mathrm{Dm}=$ effective dose, and $\mathrm{m}=$ Hill coefficient. The $\mathrm{IC}_{50}$ and the resistance index (RI) $\left[\mathrm{RI}=\mathrm{IC}_{50}\right.$ (drug-resistant cells)/IC ${ }_{50}$ (parent cells)] of HT29 and HT29/L-OHP cells to these antineoplastic drugs were calculated.

\section{Ultrastructure study}

To study the ultrastructure of tumor cells after treatment with antineoplastic drugs, the cells were fixed with $2.5 \%$ glutaric dialdehyde followed by $2 \%$ osmium tetraoxide, and then were dehydrated, embedded, sectioned, and stained as per standard protocol. The cell morphologies of HT29 and HT29/L-OHP cells were observed by a transmission electron microscope (HITACHI-600, Tokyo, Japan).

\section{Population doubling time (TD) and growth curve determination}

HT29/L-OHP and HT29 cells in logarithmic growth phase were seeded into $21 \times 15$ $\mathrm{mL}$ culture flasks at 10,000 cells/flask and cultured at $37^{\circ} \mathrm{C}$ in a $5 \% \mathrm{CO}_{2}$ cell incubator. Live cells were ascertained by trypan blue staining and counted with a hemocytometer; values were recalculated from triplicate flasks each day for a consecutive 7 days. Population TD was calculated by the Patterson formula: TD $=24 \times \mathrm{T} \times \log 2 /(\log \mathrm{Nt}-\log \mathrm{N} 0)$, where N0 is the initial number of cells, $\mathrm{Nt}$ is the final number of cells, and $\mathrm{T}$ is the culture time.

\section{Reverse transcription-polymerase chain reaction (RT-PCR) analysis}

To assess tsg101 mRNA expression, the total RNAs of HT29/L-OHP and HT29 cells were extracted, cDNA was synthesized (Invitrogen, USA), and an equal amount was 
used for PCR amplification for each sample condition. The primers for tsg101 were designed based on the GenBank (GI: 1772663) sequence as follows: Primer 1 sequences: sense: 5'ATC CCT GTG CCT TAT AGA GG-3', antisense: 5'-AAC GGG TAA CCA TCT CTT CC-3', PCR product length, 654 bp. Primer 2 sequences: sense: 5'-TTA ACG ATG GCA GTT CCA GG-3', antisense: 5'-TGA GAA CTT GTT GTG GCA GG-3', PCR product length, $490 \mathrm{bp}$. The primers for the control GAPDH gene were designed based on GenBank (GI: 83641890) sequence as follows: Primer sequences: sense: 5'-CGA CCA CTT TGT CAA GCT CA-3', antisense: 5'-AGG GGT CTA CAT GGC AAC TG-3', PCR product length, $223 \mathrm{bp}$. The samples were divided into two groups, HT29 and HT29/L-OHP. The experiments were performed according to the protocol of the Access RT-PCR System (ABI, USA). After separation by agarose gel electrophoresis, images were taken and stored in a computer for further analysis. The integral optical density (IOD) of each band was measured by the Gel-PRO ANALYZER software (Media Cybernetics, Inc., USA). The ratios between the target and control gene IODs represented the relative mRNA expression level, i.e., the standardized integral density value (normalized integrated intensity, NII).

\section{Western blotting analysis}

To measure TSG101 protein expression, the total protein contents of HT29 and HT29/L-OHP cells were isolated with $300 \mu \mathrm{L}$ RIPA solution. The protein samples were boiled for $5 \mathrm{~min}$ before measurement. Aliquots of $20 \mu \mathrm{g}$ proteins were loaded onto and separated by polyacrylamide gel electrophoresis; the proteins were subsequently transferred to a nitrocellulose membrane. The membrane was blocked using 5\% dry milk in phosphatebuffered saline (PBS) for $1 \mathrm{~h}$, and then probed with specific primary antibodies (1:1000). After washing off the primary antibody with PBS, the membrane was incubated with specific secondary antibodies conjugated with peroxidase. Enhanced chemiluminescence (BIO-RAD) was used to develop the signals, which were quantified by a Gel Doc XP Imaging System (BIO-RAD) and further corrected by the removal of background signals.

\section{Immunocytochemical analysis}

HT29 and HT29/L-OHP cells growing at logarithmic phase were harvested and digested with pancreatic enzyme (Hyclone, Logan, UT, USA); trypsin $0.25 \%$ (1X) solution + EDTA-calcium-magnesium) for 2 min into a single-cell solution at $1 \times 10^{4}$ cells $/ \mathrm{mL}$. The cells $\left(1 \times 10^{4}\right)$ were incubated on a 24 -well plate at $37^{\circ} \mathrm{C}$ and $5 \% \mathrm{CO}_{2}$ for 3 days. Then, the cells were washed twice by PBS, and fixed with $0.3 \% \mathrm{H}_{2} \mathrm{O}_{2}$-methanol solution at $37^{\circ} \mathrm{C}$ for $20 \mathrm{~min}$ to eliminate endogenous peroxidase. Cells were washed in PBS 3 times for 5 min each time, and then were blocked with normal sheep blood serum at $37^{\circ} \mathrm{C}$ for 20 min. The cells were subsequently incubated with antibodies specific to gene and protein markers, i.e., MRP, GST- $\pi$, NF-kB, HSP70, iNOS, MTP53, Bcl-2, or Bax $(1: 1000)$ at $4^{\circ} \mathrm{C}$ in a moist chamber overnight. PBS was used as a negative control for the 1st antibody. The next day, the antibody solutions were washed away by PBS, and anti-mouse/rabbit secondary antibody was applied and incubated with the cells for $30 \mathrm{~min}$ at $37^{\circ} \mathrm{C}$. After another PBS wash, the $3,3^{\prime}$-diaminobenzidine was applied and the cells were incubated at $37^{\circ} \mathrm{C}$ for $30 \mathrm{~min}$. Color development was achieved by application of freshly made 3,3'-diaminobenzidine with incubation at $37^{\circ} \mathrm{C}$ for $1-5 \mathrm{~min}$, with monitoring under a microscope; the developed cells were 
then air dried. The slides were sealed and observed under a light microscope. Pictures were taken and analyzed by the biomedical image software program CM-2000B, provided by the Beijing University of Aeronautics and Astronautics (Beijing, China). Five representative fields each were selected from the control and experimental groups under 200X magnification. The light density values were determined and the average light density values were calculated, which represented the expression levels of the proteins tested.

\section{Statistical analysis}

Statistical analysis was performed using the SPSS11.0 software (SPSS, Chicago, IL, USA). Data are reported as means \pm standard deviation. Statistical analysis was performed with an independent $t$-test. $\mathrm{P}<0.05$ was considered to be statistically significant.

\section{RESULTS}

\section{Establishment of the HT29/L-OHP cell line with stable drug resistance}

After 3 months of exposure to gradually increasing doses of L-OHP using intermittent administration to HTP29 cells in vitro, the HT29/L-OHP drug-resistant cell line was successfully established that grew well in medium containing $15 \mu \mathrm{M} \mathrm{L-OHP.} \mathrm{An} \mathrm{MTT} \mathrm{assay}$ revealed that the $\mathrm{IC}_{50}$ of the HT29 cell line was $11.9543 \mu \mathrm{M}$, whereas the $\mathrm{IC}_{50}$ of the HT29/LOHP cell line was $127.2292 \mu \mathrm{M}$ and the RI was 10.6430. Furthermore, the $\mathrm{IC}_{50}$ of the HT29/LOHP cell line was $115.1199 \mu \mathrm{M}$, and the RI was 9.6300 even following incubation without $\mathrm{L}-\mathrm{OHP}$ for 1 month. After 3 months under the maintenance drug conditions, the $\mathrm{IC}_{50}$ of the HT29/L-OHP cell line was $125.9027 \mu \mathrm{M}$ and the RI was 10.5320 .

\section{Tumor cell ultrastructure}

Under transmission electron microscopy, the HT29 cells showed irregular cell nuclei, a well-developed chromatin structure, and cell surfaces enriched in microvilli (Figure 1A). In contrast, the HT29/L-OHP cells had larger nuclei with more nuclear morbidity, visible falseinclusion bodies, a well-developed chromatin structure, fewer microvilli on the cell surfaces, and moderate expansion within the cytoplasmic rough endoplasmic reticulum (Figure 1B).

A

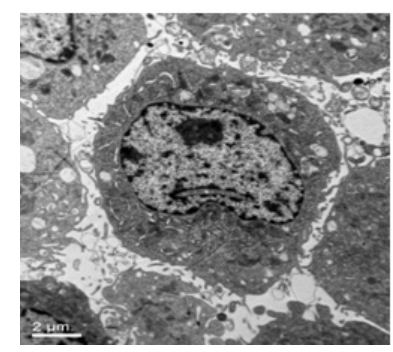

B

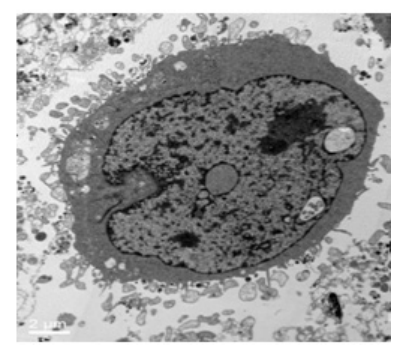

Figure 1. Tumor cell morphology ultrastructure observed with transmission electron microscopy. A. HT29 cell. B. HT29/L-OHP cell. 


\section{Cell growth curves and population TDs}

The population TDs for HT29 and HT29/L-OHP cells were 30.1 and $59.8 \mathrm{~h}$, respectively, and the difference was statistically significant $(\mathrm{P}<0.01)$. The population $T D$ was $29.7 \mathrm{~h}$ longer in the drug-resistant cells than in the parental cells (Figure 2).

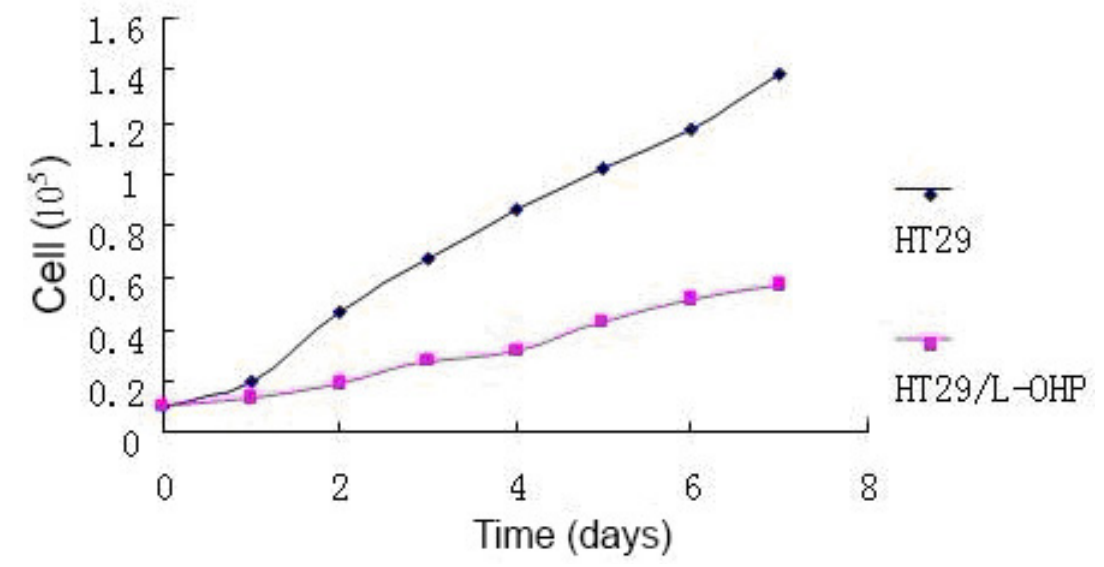

Figure 2. Growth curves of HT29 and HT29/L-OHP cells. HT29 cells: y $=0.1871 x+0.0805$, R2 = 0.9939; and HT29/L-OHP cells: $\mathrm{y}=0.0704 \mathrm{x}+0.0697, \mathrm{R} 2=0.9846$.

\section{MDR of the HT29/L-OHP cell line}

The HT29/L-OHP cell line was resistant to L-OHP as well as to four other common antineoplastic drugs to various degrees (Table 1). Thus, the HT29/L-OHP cell line constituted an MDR cancer cell line, showing the typical MDR phenotype, and might therefore serve as an ideal model for studying the mechanisms of resistance to L-OHP and filtrating reversing drug.

Table 1. Drug sensitivity of $\mathrm{HT} 29$ and HT29/L-OHP cells.
\begin{tabular}{lcrr}
\hline Drug & \multicolumn{2}{c}{$\mathrm{IC}_{50}(\mu \mathrm{M})$} & $\mathrm{RI}$ \\
\cline { 2 - 4 } & $\mathrm{HT} 29$ & $\mathrm{HT} 29 / \mathrm{L}-\mathrm{OHP}$ & 10.6430 \\
\hline $\mathrm{L}-\mathrm{OHP}$ & 11.9543 & 127.2292 & 7.1667 \\
VCR & 0.6000 & 4.3000 & 6.2274 \\
5-FU & 364.5000 & 2269.9000 & 5.6224 \\
$\mathrm{ADM}$ & 8.0003 & 44.9812 & 2.8547 \\
MMC & 1.6519 & 4.7156 & \\
\hline IC & 50 half maximal inhibitory concentration; RI = resistance index; L-OHP = oxaliplatin; VCR = vincristine; 5-FU \\
= fluorouracil; ADM = doxorubicin; MMC = mitomycin.
\end{tabular}

\section{Analysis of drug resistance-related gene expression}

The expression levels of several genes related to drug resistance were examined by immunocytochemical staining. The results of immunocytochemical analysis indicated that there was a significant increase in MRP, GST- $\pi$, NF-kB, HSP70, iNOS, MTP53, and Bcl-2 
expression in the HT29/L-OHP cell line compared to its parent line $(\mathrm{P}<0.05$; Table 2 and Figure 3). During the occurrence of membrane-mediated tumor MDR, MRP expression might alter the cellular electric potential and $\mathrm{pH}$, and block drugs from reaching their cellular target sites, thus causing drug resistance (Keppler et al., 2000). Studies have shown that MRP might function in conjunction with glutathione-s-transferase- $\pi$ (GST- $\pi$ ). GST- $\pi$ promotes glutathione (GSH) to form a complex with the anti-cancer compound; subsequently, MRP then transports the GSH-drug complex out of the cell with the result that the cell exhibits resistance to the drugs (Mulder et al., 1995). In this study, we observed an elevated level of MRP expression in HT29/L-OHP cells compared to that in its parental cell line, implying a role for MRP in the mechanism of MDR (Yakirevich et al., 2006).

Table 2. Immunohistochemical analysis results for HT29 and HT29/L-OHP cells.

\begin{tabular}{lcccccccc}
\hline & MRP & GST- $\pi$ & HSP70 & NF-kB & iNOS & MTP53 & Bcl- 2 & Bax \\
\hline HT29 & $0.1940 \pm 0.0150$ & $0.1933 \pm 0.0236$ & $0.7150 \pm 0.1052$ & $0.2950 \pm 0.0439$ & $0.2900 \pm 0.0223$ & $1.0700 \pm 0.0712$ & $0.2975 \pm 0.0432$ & $0.0047 \pm 0.0256$ \\
HT29/L-OHP & $0.1300 \pm 0.0341$ & $0.1200 \pm 0.0294$ & $0.3675 \pm 0.0432$ & $0.2175 \pm 0.0259$ & $0.1425 \pm 0.0192$ & $0.6333 \pm 0.0339$ & $0.1520 \pm 0.0084$ & $0.0048 \pm 0.0045$ \\
$T$ & 5.9813 & 10.3286 & 8.7092 & 5.000 & 22.6923 & 4.9174 & 11.3241 & 0.3458 \\
P & $<0.01$ & $<0.01$ & $<0.01$ & $<0.01$ & $<0.01$ & $<0.01$ & $<0.01$ & $>0.05$ \\
\hline
\end{tabular}

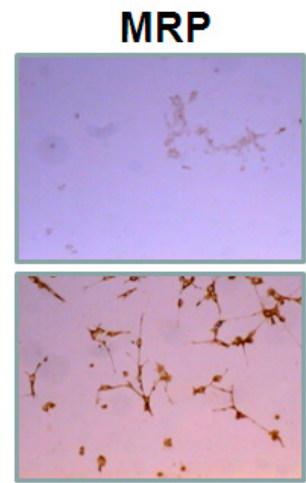

HSP70
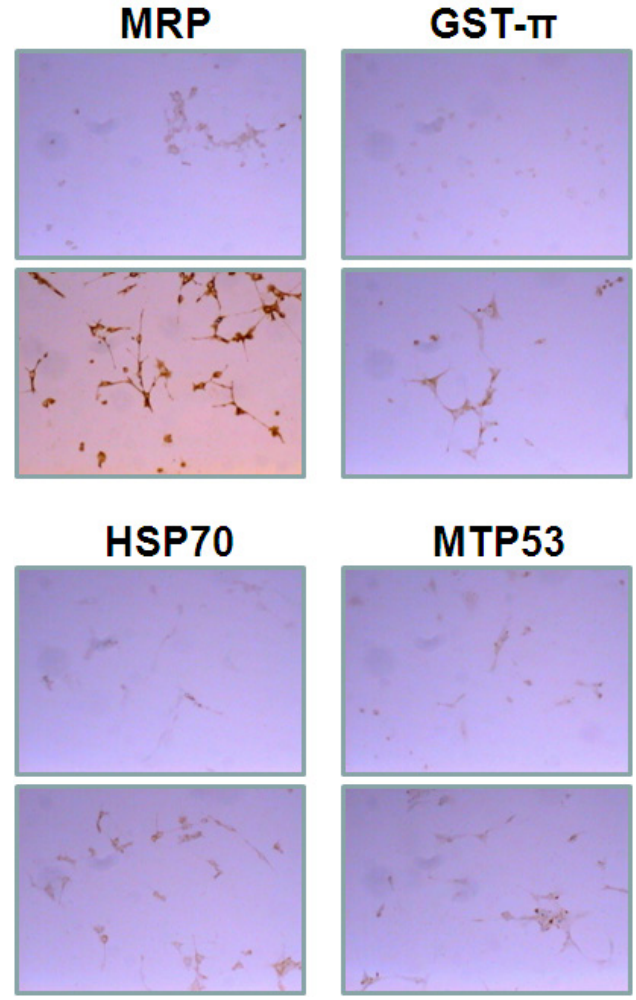

MTP53
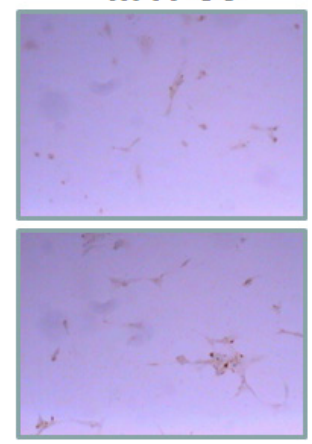

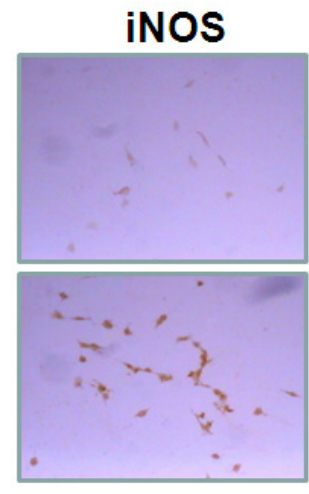

Bax
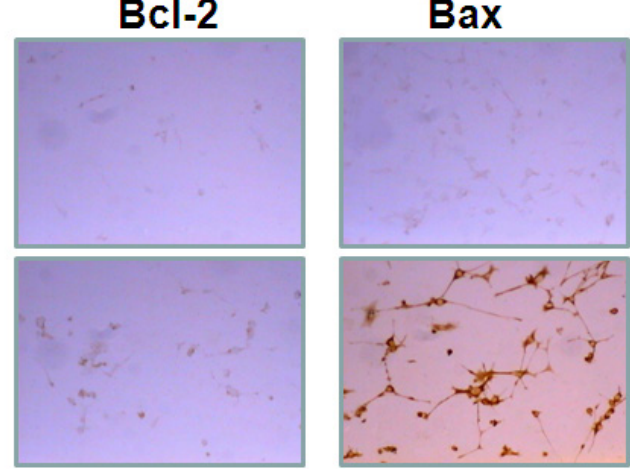

Figure 3. Immunocytochemical analysis. HT29 and HT29/L-OHP cells were immunostained for MRP, GST- $\pi$, NF$\kappa \mathrm{B}, \mathrm{HSP} 70$, iNOS, MTP53, Bcl-2, and Bax. 


\section{TSG101 expression}

TSG101 was identified in tumor cells as a functional protein. We therefore also checked its differential expressions between the two cell lines. The ratios between the target and control gene IOD represented the level of target gene mRNA expression, presented as the standardized integral light density value (NII). We calculated NII x $100 \%$, took NII\% as the unit, and compared these data with the RT-PCR results. For HT29 cells $(\mathrm{N}=3)$ (average of 3 experiments), the NII was $10.27 \pm 1.13$; and for HT29/L-OHP $(\mathrm{N}=3)$ (average of 3 experiments), the NII was $29.26 \pm 2.87$. The difference between HT29 and HT29/L-OHP NIIs was statistically significant $(\mathrm{P}<0.05$; Figure $4 \mathrm{~A})$. TSG101 protein expression as determined by western blotting is presented in Figure 4B. The HT29 cell intensity $(\mathrm{N}=3)$ (average of 3 experiments) was $15634 \pm 256$; and the HT29/L-OHP cell intensity $(\mathrm{N}=3)$ (average of 3 experiments) was $50127 \pm 315$. The results indicate that the TSG101 protein level was significantly lower in HT29 cells than in HT29/L-OHP cells $(\mathrm{P}<0.05)$.

$\mathbf{A}$

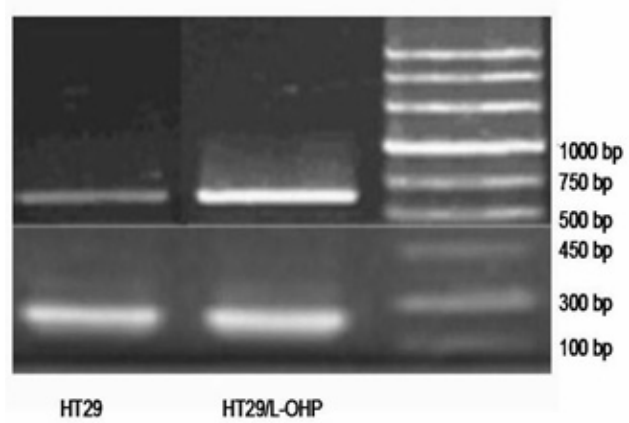

B

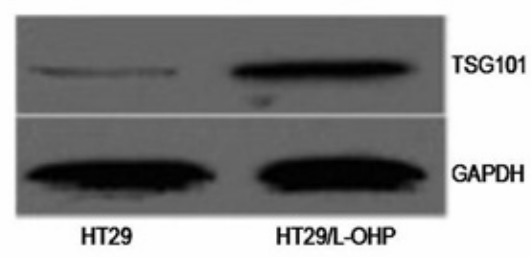

HT29
HT29/L-OHP

Figure 4. Expression of TSG101. A. TSG101 mRNA detected by RT-PCR. B. TSG101 protein detected by westernblot analysis. RT-PCR $=$ reverse transcription-polymerase chain reaction.

\section{DISCUSSION}

Colorectal cancer is a major cause of cancer-related mortality worldwide. The treatment of colorectal cancer depends on its stage. When colorectal cancer is caught early surgery can be curative. Chemotherapy may be used in addition to surgery in certain cases as an adjuvant therapy. However, drug resistance or even MDR represents a major obstacle for chemotherapeutic treatment of colorectal cancers.

GST is an enzyme involved in enzyme-mediated MDR. It comprises an intracellular detoxifying system, which promotes the transformation and metabolism of drugs, reducing their cytotoxic effects. Many anticancer drugs induce apoptosis via this action, in turn, evasion of apoptosis is a key mechanism by which tumor cells develop drug resistance. Apoptosismediated MDR involves the expression of factors such as bcl-2, Bax, NF-kB, iNOS, HSP70, and MTP53 (Anto et al., 2002). tsg101 was recently identified as a tumor-suppressing gene. 
Expression of TSG101 was observed in tumor cells as a multifunction protein (Li and Cohen, 1996). Shen et al. (2004) constructed a specific $t s g 101$ siRNA that they used to transfect gastric cancer SGC-7901 cells, which resulted in resistant to vincristine. When TSG101 expression was sharply inhibited, anticancer drugs tended to enhance the sensitivity to Changchun new alkali and to doxorubicin, implying an important role for the $t s g 101$ gene in MDR.

The recently introduced concept that cancer stem cells (CSCs) drive tumor growth suggests a reason for these therapeutic failures (Boman and Huang, 2008). Some evidence suggested that anti-metabolite cancer drugs can lead to propagation of CSCs (Yi et al., 2012). Accordingly, chemotherapy drug-induced tumor cells might be an effective way to obtain CSC-like cells. Based on this concept, we established the HT29/L-OHP cell line. In our opinion, the HP29/L-OHP cell line and CSCs share common features.

One characteristic of CSCs that differentiates them from other normal tumor cells is that they have high levels of $\mathrm{ABC}$ transporter proteins, in particular ABCG2. In clinical practice, optimal chemotherapy treatment can kill most cells within solid tumors. However, a small fraction of cells (postulated to be CSCs) are drug resistant, possibly because of enrichment of $\mathrm{ABC}$ transporter proteins and the resultant rapid and effective efflux of drugs out of the cells. Over a period of time and due to stimuli (e.g., release of cytokines or expression of heat-shock proteins) associated with tumor cell death, these quiescent stem cells are induced to divide and to produce progenitor cells. Subsequently, some of these progenitor cells differentiate into new mature tumor cells with a chemoresistant phenotype.

In addition, stem cells express pathways such as NF- $\mathrm{kB}$ and bcl-2 that protect them from the induction of apoptosis (Turco et al., 2004). In this study, the resistance to L-OHP in the human colon cancer HT29/L-OHP cell line was correlated with a high expression of MRP, GST- $\pi$, NF- $\kappa$ B, iNOS, HSP70, MTP53, Bcl-2, and TSG101 in these cells. GST- $\pi$ and BCL-2 family proteins have been implicated in redox regulation. Researchers have postulated that CSCs might be the "culprits" that are capable of evading conventional therapy. Thus, eradication of CSCs could lead to a complete cure from the cancer (Clarke et al., 2006).

In summary, we investigated the mechanisms underlying the MDR of the human colon cancer HT29/L-OHP cell line. Our data demonstrated that MDR is related to decreased intracellular storage of L-OHP, enhanced detoxifying action by GST, inhibited apoptosis, and increased expression of $t s g 101$. The role of other pathways (e.g., P-glycoprotein, the tumor microenvironment, LRP, BCRP, or Topo-PKC) in MDR remains unclear, as does whether similar mechanisms are responsible for MDR in CSCs This study provides a basis for further evaluation of these putative markers of oxaliplatin response and resistance, and even of MDR in patients with colorectal cancer who are candidates for treatment with oxaliplatin.

\section{Conflicts of interest}

The authors declare no conflict of interest.

\section{ACKNOWLEDGMENTS}

Research supported by the Natural Science Foundation Project of Chongqing Science and Technology Commission (\#cstc2012gg-yyjs0644). 


\section{REFERENCES}

Anto RJ, Mukhopadhyay A, Shishodia S, Gairola CG, et al. (2002). Cigarette smoke condensate activates nuclear transcription factor-kappaB through phosphorylation and degradation of IkappaB(alpha): correlation with induction of cyclooxygenase-2. Carcinogenesis 23: 1511-1518.

Boman BM and Huang E (2008). Human colon cancer stem cells: a new paradigm in gastrointestinal oncology. J. Clin. Oncol. 26: 2828-2838.

Clarke MF, Dick JE, Dirks PB, Eaves CJ, et al. (2006). Cancer stem cells - perspectives on current status and future directions: AACR Workshop on cancer stem cells. Cancer Res. 66: 9339-9344.

Keppler D, Kamisako T, Leier I, Cui Y, et al. (2000). Localization, substrate specificity, and drug resistance conferred by conjugate export pumps of the MRP family. Adv. Enzyme Regul. 40: 339-349.

Krishna R and Mayer LD (2000). Multidrug resistance (MDR) in cancer. Mechanisms, reversal using modulators of multidrug resistance and the role of multi-drug resistance modulators in influencing the pharmacokinetics of anticancer drugs. Eur. J. Pharm. Sci. 11: 265-283.

Li L and Cohen SN (1996). Tsg101: a novel tumor susceptibility gene isolated by controlled homozygous functional knockout of allelic loci in mammalian cells. Cell 85: 319-329.

Mulder TP, Verspaget HW, Sier CF, Roelofs HM, et al. (1995). Glutathione S-transferase pi in colorectal tumours is predictive for overall survival. Cancer Res. 55: 2696-2702.

Shen H, Pan Y, Han Z, Hong L, et al. (2004). Reversal of multidrug resistance of gastric cancer cells by downregulation of TSG101 with TSG101siRNA. Cancer Biol. Ther. 3: 561-565.

Turco MC, Romano MF, Petrella A, Bisogni R, et al. (2004). NF-kappaB/Rel-mediated regulation of apoptosis in hematologic malignancies and normal hematopoietic progenitors. Leukemia 18: 11-17.

Voland C, Bord A, Péleraux A, Pénarier G, et al. (2006). Repression of cell cycle-related proteins by oxaliplatin but not cisplatin in human colon cancer cells. Mol. Cancer Ther. 5: 2149-2157.

Yakirevich E, Sabo E, Naroditsky I, Sova Y, et al. (2006). Multidrug resistance-related phenotype and apoptosis-related protein expression in ovarian serous carcinomas. Gynecol. Oncol. 100: 152-159.

Yi H, Cho HJ, Cho SM, Jo K, et al. (2012). Effect of 5-FU and MTX on the expression of drug-resistance related cancer stem cell markers in non-small cell lung cancer cells. Korean J. Physiol. Pharmacol. 16: 11-16. 